\title{
Introduction to the Special Issue on Family Therapy with Adolescents in Residential Treatment
}

\author{
Jacob D. Christenson ${ }^{1}$
}

Published online: 10 February 2016

(c) Springer Science+Business Media New York 2016

Most counselors and therapists would agree that engaging the whole family and working with the entire system is the preferred approach; nevertheless, families with a troubled adolescent or young adult may find themselves in a position where outpatient treatment has failed and they are in need of additional support. When this is the case, families and social agencies may turn to inpatient treatment to address contributing factors. Adolescents and young adults in residential treatment present with significant mental health concerns, including conduct disorder, oppositional behavior, drug and alcohol abuse, and eating disorders, to name a few. Parents may fear that without some type of out-of-home placement the child will end up in a psychiatric hospital, or worse, incarcerated. Even though the problems may be severe, making the choice to send a child away to a program is often a heart wrenching decision and parents may be susceptible to guilt as a result (Frensch and Cameron 2002).

Families will need support from therapists at home and in the program to help them navigate the challenges that will arise while their child is placed in treatment. Although there is some disagreement concerning what constitutes residential treatment (e.g., Lee and Barth 2011) parents are able to choose from a variety of programs with different structures, purposes, and methods. Among the options are emotional growth schools, therapeutic boarding schools, wilderness therapy programs, and residential treatment centers. Given the various approaches to treatment, it is important for parents to research and understand the

Jacob D. Christenson

jchristenson@mtmercy.edu

1 Marriage and Family Therapy, Mount Mercy University, 1330 Elmhurst Dr. NE, Cedar Rapids, IA 52402, USA different options. When this process is overwhelming, it may be helpful to enlist the help of an Educational Consultant (EC) who specializes in matching individuals with treatment options.

While the child is in treatment, therapists within the program will be tasked with engaging the family in therapeutic activities. For some time now it has been recognized that children do better in residential care when their family is involved in the treatment process (e.g., Jenson and Whittaker 1987). Accordingly, it is now recognized within the industry that programs must have family involvement and work to modify the home environment that the child will eventually return to after discharge. Although this is understood, the separation between the child and parents makes it difficult to deliver traditional family therapy. This creates unique challenges for practitioners working in these settings. They must become proficient in working directly with the individual in treatment, as well as with the family, both directly and indirectly. Often family members are hundreds of miles away and may only be available by phone or for brief family visits a few times during the entire course of the program. Even when families live near the program, practitioners may find there are other barriers, such as a lack of motivation, abandonment of the child, and lengthy stays in residential programs (Burns et al. 1999) that make conjoint treatment difficult. Family therapists are often required to be creative and find unique ways of engaging the family. Once treatment is completed in a particular program, aftercare plans must be created and families will need help in determining what type of follow-up care is needed and how to modify family life to regulate the environment for the returning adolescent.

Once interventions and models are developed, the need to establish the effectiveness of those interventions and 
models becomes apparent. Historically, the research in this field has been criticized for being limited to descriptive data or correlational analysis, without reliable measures or longitudinal data (e.g., Hattie et al. 1997). Although more rigorous methods have been used more recently, the majority of the research continues to be concerned with program evaluation focused on the individual, rather than on determining what specific assessment methods and treatments are effective with this population (McLendon et al. 2012). This leaves the field vulnerable to reproach from those who see residential placements as less effective and ignores the importance of the family in the process. Accordingly, more research is needed to strengthen the place of family therapy in residential settings for adolescents and young adults.

In order to further the conversation about techniques and research, this special issue will focus on family therapy with adolescents in residential settings. One of the articles reviews in depth the available research and demonstrates some of the significant gaps that remain in the literature. One article specifically focuses on explicating research methods that can be used in these settings to further strengthen the place of family therapy. This article provides a number of ideas for how researchers can establish both the clinical and cost effectiveness of family therapy. Three of the articles report findings from research focused on intervention with adolescents in residential settings. The first focuses on how family communication is enhanced by participating in a wilderness experience. Another article reports on the coping strategies adolescents use in treatment and offers practical suggestions for how therapists can use the information to improve services. Finally, an article is included that reveals the effect of clinical consultation on outcomes.

In addition to research focused articles, the special issue includes a number of articles designed to helping practitioners better understand how to work productively in these settings. As noted above, practitioners may come out of traditional training programs with little understanding about how to work with families who are separated by distance or circumstances. A few articles were included that speak about working with this population in general. One of these articles discusses how to effectively use letter writing in a therapeutic manner to improve the relationship between the parent and child. Another focuses on the Stages of Change model (Prochaska et al. 1994) as a framework for increasing motivation to change in the identified patient and the family as a whole.

There were also five additional articles that discuss intervention and treatment techniques within a wilderness setting (also known as Outdoor Behavioral Healthcare). The use of Outdoor Behavioral Healthcare (OBH) as a high impact intervention for adolescents and young adults has grown over the last few decades, and it is exciting to be able to include information garnered from such programs. One of these articles reports on the use of Narrative Family Therapy with an adolescent in an OBH program and provides detailed information on how to replicate the author's efforts. There is an article that provides information on the role of family therapy in $\mathrm{OBH}$ and another that describes how family sculptures have been modified for use when families are separated. Another article is a modified case study describing the experience of families who have participated in an OBH program. This article also offers information about the role of an EC and provides practical suggestions that families and providers can use. Finally, an article was included that discusses the role of aftercare following discharge from an OBH program.

Overall, the articles included in this special issue provide detailed information, beyond what is available in the literature currently, about how to work with adolescents in residential placements. Those who read these articles will gain a greater understanding of the importance of research in establishing the place of family therapy in these settings and improve their ability to deliver services. As always, the information presented herein should be seen as a foundation that can be used to further inquiry and research focused on these settings.

\section{References}

Burns, B. J., Hoagwood, K., \& Mrazek, P. (1999). Effective treatment for mental disorders in children and adolescents. Clinical Child and Family Psychology Review, 2, 199-254.

Frensch, K. M., \& Cameron, G. (2002). Treatment of choice or a last resort? A review of residential mental health placements for children and youth. Child \& Youth Care Forum, 31(5), 307-339. doi:10.1023/A:1016826627406.

Hattie, J., Marsh, H. W., Neill, J. T., \& Richards, G. E. (1997). Adventure education and outward bound: Out-of-class experiences that make a lasting difference. Review of Educational Research, 67(1), 43-87. doi:10.3102/00346543067001043.

Jenson, J. M., \& Whittaker, J. K. (1987). Parental involvement in children's residential treatment. Children and Youth Services Review, 9, 81-100. doi:10.1016/0190-7409(87)90011-9.

Lee, B. R., \& Barth, R. P. (2011). Defining group care programs: An index of reporting standards. Child \& Youth Care Forum, 40, 253-266. doi:10.1007/s10566-011-9143-9.

McLendon, T., McLendon, D., \& Hatch, L. (2012). Engaging families in the residential treatment process utilizing family-directed structural therapy. Residential Treatment for Children \& Youth, 29(1), 66-77.

Prochaska, J. O., Norcross, J. C., \& DiClemente, C. C. (1994). Changing for good: A revolutionary six-stage program for overcoming bad habits and moving your life forward. New York, NY: HarperCollins. 